\title{
Effet de l'addition de méthionine protégée à la ration des vaches laitières: influence du niveau des apports azotés
}

\author{
B. REMOND \\ avec la collaboration technique de Renée Lefaivre et A. Ollier \\ INRA, Laboratoire de la Lactation, Centre de Recherches de Clermont-Ferrand-Theix \\ Saint-Genès-Champanelle, F 63122 Ceyrat
}

\begin{abstract}
Résumé
Dans 2 essais conduits pendant la phase descendante de la lactation, l'effet de la complémentation de rations de vaches laitières avec $10 \mathrm{~g}$ de $\mathrm{D}, \mathrm{L}$ méthionine protégée a été étudié, selon le niveau des apports azotés. Dans l'essai 1 d'une durée de 9 semaines, la ration, à base d'ensilage de mais, était offerte en quantité limitée. Les 25 vaches primipares étaient réparties en 3 lots : un lot bien alimenté en azote et 2 lots sous-alimentés (respectivement 17,7 p. 100 et 13,6 p. 100 de matières azotées dans la matière sèche), dont l'un était complémenté avec de la méthionine. Dans l'essai 2 d'une durée de 13 semaines, le fourrage qui était constitué d'ensilage d'herbe était offert à volonté. Les 35 vaches étaient réparties en 4 lots selon un schéma factoriel $2 \times 2$ : alimentation azotée satisfaisante ou insuffisante (respectivement 17,5 et 13,6 p. 100 de MAT dans la MS) $\times$ complémentation ou non en $\mathrm{D}, \mathrm{L}$ méthionine.

L'apport de $\mathrm{D}, \mathrm{L}$ méthionine protégée a accru la méthioninémie mais n'a pas modifié les performances des animaux, ni les quantités ingérées. L'accroissement des apports azotés, à partir de niveaux insuffisants, a entraîné des augmentations des quantités d'aliments ingérées (Essai 2), de la digestibilité de la ration, de la quantité de lait produite et du gain de poids vif de respectivement $0,39 \mathrm{~kg}$ de $\mathrm{MS}, 0,60$ point de digestibilité, $0,50 \mathrm{~kg}$ et $0,05 \mathrm{~kg}$ par point $\mathrm{p} .100 \mathrm{de}$ matières azotées totales en plus dans la ration.
\end{abstract}

Mots clés: Vache laitière, méthionine, niveaux des apports azotés, lait, digestibilité.

\section{Introduction}

Depuis les travaux de Mc Carthy et al. (1968) qui ont montré le rôle de la carence en méthionine dans l'étiologie des cétoses chez les vaches laitières, de nombreux essais ont visé à rechercher l'intérêt de la complémentation des régimes de ces animaux avec la forme racémique de la molécule ( $\mathrm{D}, \mathrm{L}$ méthionine) et surtout avec son analogue hydroxylé (MHA). Quand des effets ont été constatés, ils ont très généralement concerné le taux butyreux et la sécrétion des matières grasses dans le lait (i.a. REMOND et al., 1971). Cependant, ces effets n'ont pas été systématiques (i.a. CASPER et al., 1987) et certains auteurs se sont demandé si l'efficacité de l'apport de méthionine n'était pas liée au niveau des apports azotés de la ration et à leur richesse en méthionine. Dans 
des essais où l'apport de MHA a eu un effet favorable sur une caractéristique de la production laitière et où au moins 2 niveaux d'apports azotés étaient comparés, JENNY et al. (1980), Clay \& SATter (1979) et Lundquist et al. (1983) (dans 2 essais sur 3) n'ont pas observé d'interaction avec le niveau des apports azotés alors que ChandeEr $e t$ al. (1976), Stokes et al. (1981), et Lundquist et al. (1983) (dans 1 essai sur 3) ont observé un effet plus élevé pour le niveau le plus bas des apports azotés. Norman et al. (1982) n'ont pas, non plus, observé d'interaction entre l'apport de MHA et celui des apports azotés.

Cependant, tous ces essais avaient été réalisés avec les formes non protégées de la méthionine ou du MHA. Or, ces molécules sont rapidement dégradées dans la panse (Chalupa, 1975) et on peut se demander si là n'est pas la cause des effets faibles (en général non significatifs) ou nuls enregistrés.

Nous avons voulu, dans 2 essais, étudier l'interaction entre le niveau des apports azotés et l'apport de D,L méthionine protégée. Pour accroître la probabilité de mise en évidence d'un effet de la méthionine, nous avons, dans le $2^{\mathrm{e}}$ essai, alimenté les animaux avec des aliments pauvres en méthionine.

\section{Matériel et méthodes}

\section{A. Animaux et schéma expérimental}

Dans l'essai 1, 25 vaches primipares, ayant vêlé en moyenne à 24 mois, ont été utilisées. Après une période pré-expérimentale de 3 semaines, qui a commencé au $79^{\mathrm{e}}$ jour de lactation en moyenne, elles ont été réparties en 3 lots : 1 lot bien alimenté en azote, et 2 lots sous-alimentés, dont 1 complémenté en méthionine. La période expérimentale a duré 6 semaines.

Dans l'essai 2, 35 vaches dont 5 primipares ont été utilisées. La période préexpérimentale qui a commencé le $49^{\mathrm{e}}$ jour de lactation en moyenne, a duré 4 semaines et a été suivie d'une période expérimentale de 9 semaines au cours de laquelle les vaches étaient réparties en 4 lots selon un schéma factoriel $2 \times 2: 2$ niveaux d'apports azotés croisés avec une complémentation ou non en méthionine.

Dans les 2 essais, pendant la période pré-expérimentale, on a essayé de satisfaire les besoins nutritionnels des animaux. La mise en lots des animaux à la fin de la période pré-expérimentale s'est effectuée selon les critères suivants : quantité de lait produite et composition, poids vif, date de vêlage et, dans l'essai 2, numéro de lactation. Pendant la période expérimentale, l'apport de $\mathrm{D}, \mathrm{L}$ méthionine protégée a été de $10 \mathrm{~g}$ par jour.

\section{B. Alimentation}

\section{Essai 1}

Le régime, qui avait une composition constante, était composé (sur la base de la matière sèche) de 65 p. 100 d'ensilage de maïs et de 35 p. 100 d'un mélange de tourteaux de soja-colza (1/1) tannés à l'aldéhyde formique ou de tourteau de soja 
associés à un concentré énergétique. Pendant la période pré-expérimentale, le régime a été distribué à volonté et les besoins azotés des animaux ont été satisfaits par la distribution de $1,5 \mathrm{~kg}$ de tourteaux de soja-colza tannés. Pendant la période expérimentale, les animaux ont reçu des quantités d'aliments limitées, ajustées individuellement en supposant une décroissance de la quantité de lait à 4 p. 100 de MG produite de 1,5 p. 100 par semaine. Les vaches bien alimentées en azote ont eu leurs besoins azotés satisfaits par l'apport de $2 \mathrm{~kg}$ de tourteau de soja alors que les 2 lots de vaches sousalimentées en azote n'en ont pas du tout reçu. De l'urée a systématiquement été ajoutée à la ration à raison de $150 \mathrm{~g} / \mathrm{j}$ (lot bien alimenté en azote) ou $200 \mathrm{~g} / \mathrm{j}$ (lot sousalimenté en azote), de façon à satisfaire les besoins des microbes en azote soluble.

\section{Essai 2}

La ration de base était constituée d'ensilage d'herbe, supposé satisfaire les besoins énergétiques d'entretien et d'une production de $13 \mathrm{~kg}$ de lait à $4 \mathrm{p}$. $100 \mathrm{de} \mathrm{MG}$, et les besoins azotés d'entretien et d'une production de $11 \mathrm{~kg}$ de lait à $4 \mathrm{p} .100$ de MG. Ces valeurs ont été diminuées de $5 \mathrm{~kg}$ chez les primipares. Pendant la période préexpérimentale, la différence entre les besoins des animaux et les apports nutritionnels adoptés pour la ration de base a été comblée par un mélange d'un aliment concentré énergétique et d'un aliment concentré azoté ( 40 p. 100 de farine de sang et 60 p. 100 de tourteau de soja). Pendant la période expérimentale, les quantités d'énergie apportées par le concentré ont été diminuées toutes les 2 semaines en supposant une réduction de la production de lait à 4 p. 100 de matières grasses de 2,2 p. 100 par semaine chez les multipares, et de 1,5 p. 100 chez les primipares. Les apports azotés ont été ajustés individuellement (en modifiant le rapport concentré énergétique / concentré riche en protéines) de façon à largement satisfaire les besoins (55 g de PDI/ $\mathrm{kg}$ de lait à $4 \mathrm{p} .100$ de M.G.) dans les 2 lots bien alimentés en azote, et à n'apporter que $45 \mathrm{~g}$ dans les 2 lots sous-alimentés.

La D,L méthionine protégée utilisée était un produit expérimental spécialement préparé par l'AEC Rhône-Poulenc. Elle était incluse à raison de 2 p. 100 dans un mélange de céréales. Les animaux témoins ont reçu la même quantité d'aliment contenant la substance servant à protéger la méthionine. Tous les aliments ont été distribués 2 fois par jour, en mélange. Leur composition est présentée au tableau 1.

\section{Mesures}

Toutes les mesures ont été individuelles. Les quantités d'aliments distribuées ont été mesurées tous les jours, les quantités d'aliments refusées 4 fois (essai 1) ou 3 fois (essai 2) par semaine ; les quantités de lait produites ainsi que les teneurs en matières grasses et en protéines (méthode infrarouge) 4 fois (essai 1) ou 3 fois par semaine ; le poids vif, chaque semaine ou toutes les 2 semaines (essai 2). Juste après l'essai 1 et au cours de la dernière semaine de l'essai 2 , la digestibilité des régimes bien pourvus et insuffisamment pourvus en azote a été mesurée sur 4 ou 5 vaches par régime, par collecte totale des fèces pendant 5 jours. Dans l'essai 2, pour pouvoir interpréter plus facilement les résultats, la proportion d'ensilage d'herbe avait été rendue identique pour toutes les vaches : 70 p. 100 dans la matière sèche. 
TABLEAU 1

Composition chimique des aliments.

Chemical composition of foods.

\begin{tabular}{|c|c|c|c|}
\hline \multirow[t]{2}{*}{$\begin{array}{l}\text { Aliments } \\
\text { Foods }\end{array}$} & $\begin{array}{c}\text { Matière } \\
\text { sèche } \\
\% \text { aliment } \\
\text { Dry } \\
\text { matter } \\
\% \text { food }\end{array}$ & $\begin{array}{l}\text { Matière } \\
\text { organique } \\
\text { Organic } \\
\text { matter }\end{array}$ & $\begin{array}{c}\text { Matières } \\
\text { azotées } \\
\text { totales } \\
(\mathrm{N} \times 6,25) \\
\text { Crude } \\
\text { protein } \\
(N \times 6.25)\end{array}$ \\
\hline & \multicolumn{3}{|c|}{$\begin{array}{c}\text { (\% de la matière sèche) } \\
(\% \text { dry matter })\end{array}$} \\
\hline \multicolumn{4}{|l|}{ ESSAI $1-T R I A L I$} \\
\hline $\begin{array}{l}\text { Ensilage de maïs } \ldots \ldots \ldots \ldots \ldots \ldots \ldots \ldots \ldots \ldots \ldots \ldots \\
\text { Maize silage }\end{array}$ & 35,4 & 94,3 & 9,2 \\
\hline $\begin{array}{l}\text { Tourteaux de soja et de colza tannés }(1 / 1) \ldots \ldots \\
\text { Soyabean and rapeseed }(1 / 1) \text { protected meals }\end{array}$ & 88,3 & 92,5 & 46,1 \\
\hline $\begin{array}{l}\text { Tourteau de soja } \ldots \ldots \ldots \ldots \ldots \ldots \ldots \ldots \ldots \ldots \ldots \ldots \ldots \\
\text { Soyabean meal }\end{array}$ & 88,6 & 92,7 & 50,4 \\
\hline $\begin{array}{l}\text { Concentré énergétique }{ }^{(1)} \ldots \ldots \ldots \ldots \ldots \ldots \ldots \ldots \\
\text { Energy-rich concentrate }\end{array}$ & 88,2 & 92,9 & 10,9 \\
\hline $\begin{array}{l}\text { Complément minéral }{ }^{(2)} \ldots \ldots \ldots \ldots \ldots \ldots \ldots \\
\text { Mineral-rich concentrate }\end{array} \ldots \ldots \ldots \ldots$ & 95,8 & 28,6 & 2,5 \\
\hline 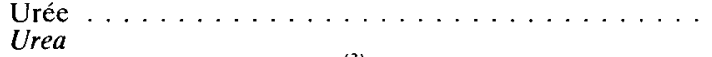 & 99,8 & 100,0 & 288,8 \\
\hline $\begin{array}{l}\text { Concentrés expérimentaux }{ }^{(3)} \ldots \ldots \ldots \ldots \ldots \ldots \ldots \\
\text { Experimental concentrates }\end{array}$ & 88,5 & 96,0 & 10,6 \\
\hline \multicolumn{4}{|l|}{ ESSAI $2-T R I A L 2$} \\
\hline $\begin{array}{l}\text { Ensilage d'herbe }{ }^{(4)} \ldots \ldots \ldots \ldots \ldots \ldots \ldots \ldots \ldots \ldots \\
\text { Grass silage }\end{array}$ & 23,2 & 91,9 & 13,2 \\
\hline $\begin{array}{l}\text { Tourteau de soja - Farine de sang }(60 / 40) \ldots \ldots \\
\text { Soyabean meal - Blood meal }(60 / 40)\end{array}$ & 88,8 & 94,1 & 70,4 \\
\hline $\begin{array}{l}\text { Concentré énergétique }{ }^{(5)} \ldots \ldots \ldots \ldots \ldots \ldots \ldots \ldots \ldots \\
\text { Energy-rich concentrate }\end{array}$ & 88,6 & 92,3 & 13,6 \\
\hline $\begin{array}{l}\text { Complément minéral }{ }^{(6)} \\
\text { Mineral-rich concentrate }\end{array}$ & 93,4 & 20,5 & 0,4 \\
\hline $\begin{array}{l}\text { Concentrés expérimentaux }{ }^{(7)} \ldots \ldots \ldots \ldots \ldots \ldots \ldots \\
\text { Experimental concentrates }\end{array}$ & 87,0 & 98,0 & 13,6 \\
\hline
\end{tabular}

(1) Composition centésimale : Maïs : $30 ;$ Orge : 25 ; Luzerne déshydratée : 10 ; Pulpes de betteraves déshydratées : 32 ; Carbonate de calcium : 1 ; Phosphate bicalcique : 1,5 ; Oxyde de magnésium : 0.5 . Centesimal composition : Maize : 30 ; Barley : 25 ; Dehydrated lucerne : 10 ; Dehydrated beet pulp :32; Calcium carbonate : $1 ;$ Dicalcium phosphate : 1.5 ; Magnesium oxyde: 0.5 .

(2) Composition centésimale : Phosphore : 10 ; Calcium : 20 ; Magnésium : 5 ; Oligoéléments (cuivre, fer, manganèse, zinc) et vitamines $\mathrm{A}, \mathrm{D}_{3}, \mathrm{E}$. Centesimal composition : Phosphorus : $10 ;$ Calcium :20; Magnesium :5; Trace elements (copper, iron, manganese, zinc) and vitamins $A, D_{3}, E$.

(3) Moyenne des 2 concentrés : Composition centćsimale du support : Maïs : 35 : Orge : $30 ;$ Pulpes : 35 . Mean of the 2 concentrates ; Centesimal composition of the support : Maize : 35 ; Barley : $30 ;$ Dehydrated beet pulp : 35 .

(4) Ensilage direct de prairie naturelle avec conservateur ( 31 acide formique/tonne de fourrage). Quelques caractéristiques fermentaires : $\mathrm{pH}=3,6$; Acide lactique $=107 \mathrm{~g}$ par $\mathrm{kg}$ de matière sèche ; $\mathrm{N}$ soluble $\% \mathrm{~N}$ total $=68 \mathrm{p}$. 100 . Direct silage from natural grassland with additive $(31$ formic aciditon of forage). Some fermentative characteristics $: \mathrm{pH}=3.6$, Lactic acid $=107 \mathrm{~g} / \mathrm{kg}$ dry matter Soluble $N \%$ total $N=68 \mathrm{p} .100$.

(5) Composition centésimale : Orge : 18,5 ; Blé : 18,0 ; Pulpes de betteraves déshydratées : 20,0 ; Manioc : 35,0 ; Mélasse : 4,0 ; Urée : 1,5 ; Carbonate de calcium : 1,0 ; Phosphate bicalcique : 1,5; Sel marin : 0.5 . Centesimal composition : Barley : 18.5 ; Wheat : 18.0 ; Dehydrated beet pulp : 20.0 ; Manioc : 35.0 ; Molasses : $4.0 ;$ Urea : 1.5 ; Calcium carbonate : 1.0 ; Dicalcium phosphate : 1.5 ; Salt : 0.5 .

(6) Composition centésimale : Phosphore : 14; Calcium : 14; Magnésium : 3 ; Oligoéléments (cuivre, zinc, manganèse) et vitamines. Centesimal composition : Phosphorus : $14 ;$ Calcium $: 14 ;$ Magnesium $: 3 ;$ Trace elements (copper, zinc, manganese) and vitamins.

(7) Moyenne des 2 concentrés ; Composition centésimale de l'aliment support : Orge : 50 ; Maïs : 50 . Mean of the 2 concentrates; Centesimal composition of the support : Barley : $50 ;$ Maize : 50 . 
Du sang a été prélevé dans la veine jugulaire, au début de l'après-midi à la $5^{\mathrm{e}}$ semaine expérimentale de l'essai 1 , et aux $5^{\mathrm{e}}$ et $8^{\mathrm{e}}$ semaines expérimentales de l'essai 2 . Dès le prélèvement (dans l'étable même) une quantité identique du sang de chaque vache a été placée dans un flacon contenant de l'alcool à $-18^{\circ} \mathrm{C}$ en vue du dosage des acides aminés libres selon la méthode déjà présentée par REMOND et al. (1971). Nous avons constitué les échantillons moyens en regroupant, par traitement expérimental et par semaine de prélèvement (essai 2), les échantillons individuels des vaches retenues dans le dépouillement de l'essai.

Les apports nutritionnels (UFL, PDI) ont été calculés d'après les modes de calcul et les Tables de valeur alimentaire des aliments publiés par l'INRA (INRA, 1978). Pour les valeurs PDI, nous avons corrigé les valeurs des Tables afin de tenir compte de la teneur en azote effective de nos aliments. Les besoins nutritionnels ont été calculés d'après les valeurs publiées par l'INRA (INRA, 1978).

\section{Analyse statistique des résultats}

Les résultats (moyenne des 4 dernières semaines expérimentales dans l'essai 1 , et des 6 dernières semaines dans l'essai 2) ont été soumis à des analyses de variance et de covariance (SEEBECK, 1973). Dans toutes les analyses, excepté celle des variations de poids vif, on a introduit, comme covariable, le même critère que celui analysé, mesuré pendant la période préexpérimentale ( 2 et 3 dernières semaines, pour les essais 1 et 2 respectivement).

\section{Résultats}

\section{A. Déroulement des essais et conformité au protocole}

Nous avons éliminé du dépouillement des résultats 4 vaches de l'essai 1 pour production sensiblement inférieure à celle des autres vaches, et 3 vaches de l'essai 2 pour mammites ayant fortement et irréversiblement affecté la production laitière des animaux.

Pendant la période préexpérimentale, les besoins énergétiques et azotés des animaux ont été satisfaits dans l'essai 1 , conformément au protocole, mais ils ne l'ont pas été dans l'essai 2 à cause de diminutions d'ingestion entre les toutes premières semaines de lactation (avant la période préexpérimentale) au cours desquelles les vaches recevaient de l'ensilage de maïs, et celles de la période préexpérimentale au cours de laquelle elles recevaient l'ensilage d'herbe. Pendant la période expérimentale, les niveaux d'apports azotés ont été plus élevés dans les lots bien alimentés que dans les lots sous-alimentés de $601 \mathrm{~g}$ de MAT (328 g de PDI) dans l'essai 1 , et de $837 \mathrm{~g}$ de MAT ( $467 \mathrm{~g}$ de PDI) dans l'essai 2 . Le degré de satisfaction des besoins azotés, estimé en calculant les besoins de production d'après la quantité de protéines sécrétée par le lot bien alimenté en azote, a été, pour les lots sous-alimentés et bien alimentés en azote, respectivement de 82 p. 100 et 115 p. 100 dans l'essai 1 , et 68 p. 100 et 109 p. 100 dans l'essai 2. 


\section{B. Effet de l'apport de méthionine protégée}

L'apport de $10 \mathrm{~g}$ de méthionine protégée aux animaux des lots sous-alimentés en azote a entraîné une augmentation de la méthioninémie de 25 p. 100 (essai 1) ou 50 p. 100 (essai 2), mais n'a pas eu d'effet lorsque les animaux étaient correctement alimentés (essai 2) (tabl. 2).

\section{TABLEAU 2}

Effet de l'apport alimentaire de $D, L$ méthionine protégée et du niveau des apports azotés sur la méthioninémie ( $\mathrm{mg} / 100 \mathrm{~g}$ sang).

Effect of protected $D, L$ methionine supplementation and of the level of protein supply on the blood level of methionine.

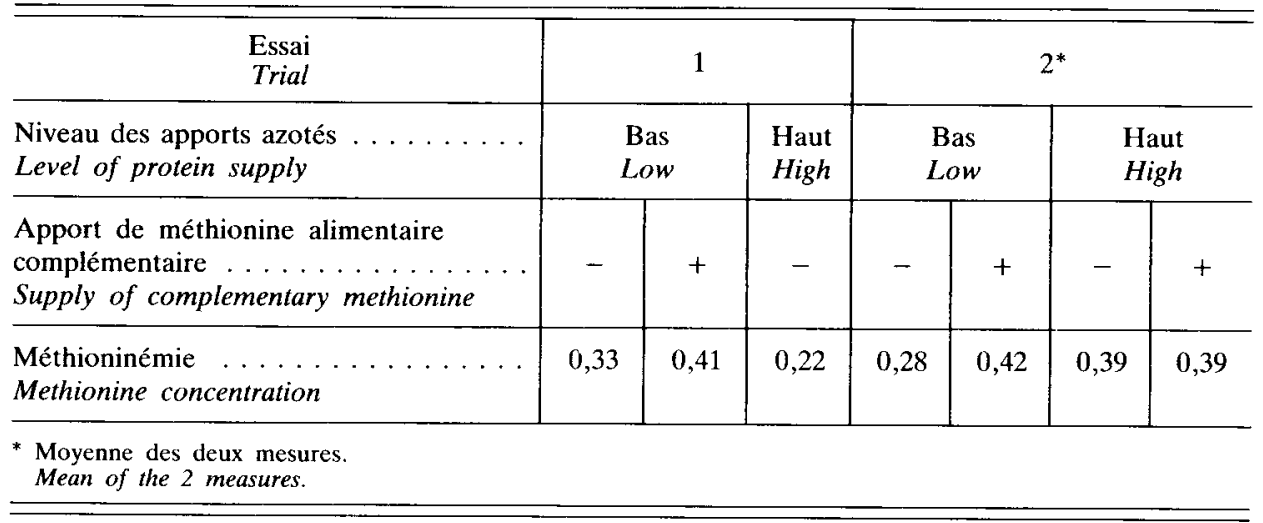

L'apport de méthionine n'a pas eu d'effet sur les quantités de lait, de matières graśses et de protéines produites, ni sur les teneurs en ces 2 constituants (tabl. 3 et 4 ). La variation de poids vif et l'efficacité de l'utilisation de la ration n'ont pas été modifiées. Dans l'essai 2 où le fourrage était offert à volonté, l'apport de méthionine protégée n’a pas non plus modifié les quantités qui en étaient ingérées.

\section{Effet du niveau des apports azotés}

L'accroissement du niveau des apports azotés a entraîné des modifications importantes des performances des animaux (tabl. 3 et 4). Les quantités d'aliments ingérées ont été accrues de $1,5 \mathrm{~kg}$ dans l'essai 2 où l'ensilage d'herbe était offert à volonté, à cause, essentiellement, de l'accroissement de l'ingestion de cet aliment. Dans les 2 essais, la digestibilité de la ration a été accrue, d'une façon beaucoup plus importante, et significative, lorsque la ration de base était constituée d'ensilage de mails que lorsqu'elle était constituée d'ensilage d'herbe (respectivement 0,95 et 0,24 point p. 100 de digestibilité de la matière organique par point p. 100 dans la matière sèche de teneur en MAT supplémentaire) (tabl. 5). Les accroissements de valeur énergétique de la ration ainsi entraînés par l'apport supplémentaire d'azote, sont de 0,016 UFL et 0,005 UFL par Kg de MS et par point de MAT (p. 100 MS) supplémentaire pour les essais 1 et 2 respectivement. 
TABleau 3

Quantités d'aliments ingérées, production laitière et bilans nutritifs au cours de l'essai 1. Food intake, milk yield and nutritive balance during trial 1.

\begin{tabular}{|c|c|c|c|}
\hline \multirow{2}{*}{$\begin{array}{c}\text { Niveau des apports azotés } \\
\text { Level of protein supply } \\
\text { Apport alimentaire de méthionine protégée } \\
\text { Supply of protected methionine }\end{array}$} & \multicolumn{2}{|c|}{$\begin{array}{l}\text { Bas } \\
\text { Low }\end{array}$} & \multirow{2}{*}{$\begin{array}{c}\text { Haut } \\
\text { High } \\
-\end{array}$} \\
\hline & - & + & \\
\hline \multicolumn{4}{|l|}{$\begin{array}{l}\text { Alimentation (kg MS/j) : } \\
\text { Feeding }(\mathrm{kg} \mathrm{DM} / \mathrm{d}) \text { : }\end{array}$} \\
\hline $\begin{array}{l}\text { Ensilage de mais } \ldots \ldots \ldots \ldots \ldots \ldots \ldots \ldots \ldots \ldots \ldots \ldots \\
\text { Maize silage }\end{array}$ & 9,18 & 8,95 & 9,39 \\
\hline $\begin{array}{l}\text { Concentré riche en énergie } \ldots \ldots \ldots \ldots \ldots \ldots \ldots \ldots \ldots \ldots \\
\text { Energy-rich concentrate }\end{array}$ & 4,42 & 4,30 & 2,54 \\
\hline $\begin{array}{l}\text { Tourteau de soja } \ldots \ldots \ldots \ldots \ldots \ldots \ldots \ldots \ldots \ldots \ldots \\
\text { Soyabean meal }\end{array}$ & - & - & 1,75 \\
\hline $\begin{array}{l}\text { Concentré expérimental } \ldots \ldots \ldots \ldots \ldots \ldots \ldots \ldots \ldots \\
\text { Experimental concentrate }\end{array} \ldots \ldots \ldots \ldots$ & 0,42 & 0,42 & 0,44 \\
\hline Urée $\ldots \ldots \ldots \ldots \ldots \ldots \ldots \ldots \ldots \ldots \ldots \ldots \ldots \ldots \ldots \ldots$ & 0,20 & 0,19 & 0,15 \\
\hline $\begin{array}{l}\text { Concentré riche en minéraux } \ldots \ldots \ldots \ldots \ldots \ldots \ldots \ldots \\
\text { Mineral-rich concentrate }\end{array}$ & 0,20 & 0,20 & 0,20 \\
\hline $\begin{array}{l}\text { Matière sèche totale ingérée } \ldots \ldots \ldots \ldots \ldots \ldots \ldots \ldots \\
\text { Total dry matter intake }\end{array}$ & 14,42 & 14,06 & 14,48 \\
\hline 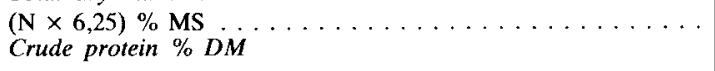 & 13,6 & 13,6 & 17,7 \\
\hline \multicolumn{4}{|l|}{$\begin{array}{l}\text { Production laitière et variation de poids vif : } \\
\text { Milk yield and liveweight variation: }\end{array}$} \\
\hline 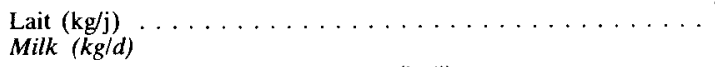 & $\begin{array}{l}17,9 \\
(-0,44 \mathrm{~B})\end{array}$ & $\left(\begin{array}{l}17,6 \\
(-0,71 \mathrm{~B})\end{array}\right.$ & $\begin{array}{c}19,7 \\
(1,15 \mathrm{~A})\end{array}$ \\
\hline $\begin{array}{l}\text { Lait à } 4 \text { p. } 100 \text { de matières grasses }(\mathrm{kg} / \mathrm{j}) \ldots \ldots \ldots \ldots \ldots \\
\text { Fat corrected milk }(F C M-k g / d)\end{array}$ & $\begin{array}{c}18,3 \\
(-0,51 \mathrm{~B})\end{array}$ & $\left(-\begin{array}{l}18,0 \\
0,56 \mathrm{~B})\end{array}\right.$ & $\begin{array}{c}19,8 \\
(1,08 \mathrm{~A})\end{array}$ \\
\hline $\begin{array}{l}\text { Taux butyreux (g p. } 1000) \ldots \ldots \ldots \ldots \\
\text { Fat content }(g \text { p. } / 000)\end{array}$ & 41,6 & 41,5 & 40,4 \\
\hline $\begin{array}{l}\text { Taux protéique (g p. } 1000) \ldots \ldots \ldots \\
\text { Protein content }(g \text { p. } 1000)\end{array}$ & $\begin{array}{l}29,8 \\
(-0,70 \mathrm{~b})\end{array}$ & $\begin{array}{l}30,0 \\
(-0,25 \mathrm{bc})\end{array}$ & $\begin{array}{c}32,0 \\
(0,96 \mathrm{ac})\end{array}$ \\
\hline $\begin{array}{l}\text { Matières grasses }(\mathrm{g} / \mathrm{j}) \ldots \ldots \\
\text { Fat }(g / d)\end{array}$ & $(-22 \mathrm{~B})$ & $(-16 \mathrm{~B})$ & $(38 \mathrm{~A})$ \\
\hline $\begin{array}{l}\text { Protéines }(g / \mathrm{j}) \quad \ldots \ldots \ldots \\
\text { Protein }(g / d)\end{array}$ & $(-26 \mathrm{~B})$ & $\begin{array}{c}524 \\
(-28 \mathrm{~B})\end{array}$ & $\begin{array}{c}627 \\
(54 \mathrm{~A})\end{array}$ \\
\hline $\begin{array}{l}\text { Variation du poids vif } \\
\text { (Période expérimentale - Période pré-expérimentale) }(\mathrm{kg}) \ldots \\
\text { Liveweight variation } \\
\text { (Experimental - pre-experimental period) }(\mathrm{kg})\end{array}$ & $\begin{array}{c}-6 \\
(-4,3 \mathrm{Bb})\end{array}$ & $(-0,8 b)$ & $\begin{array}{c}3 \\
(5,1 \mathrm{Aa})\end{array}$ \\
\hline \multicolumn{4}{|l|}{$\begin{array}{l}\text { Apports nutritionnels et efficacité alimentaire : } \\
\text { Nutritive supply and feed efficiency : }\end{array}$} \\
\hline $\begin{array}{l}\text { Apports énergétiques }(\mathrm{UFL} / \mathrm{j}) \\
\text { Energy supply }(F U m / d)\end{array}$ & 11,92 & 11,65 & 12,99 \\
\hline 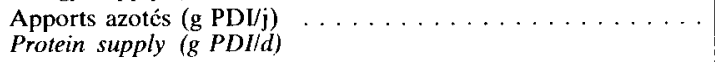 & 1179 & 1148 & 1491 \\
\hline $\begin{array}{l}\text { Lait } 4 \text { p. } 100 \mathrm{MG} / \mathrm{UFL} \text { disponible pour la production } \ldots \ldots \\
\text { FCM/FUm for milk yield }\end{array}$ & $\begin{array}{c}2,53 \\
(0,06 \mathrm{~B})\end{array}$ & $\begin{array}{c}2,52 \\
(0,05 \mathrm{~B})\end{array}$ & 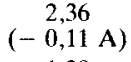 \\
\hline $\begin{array}{l}\text { Lait } 4 \text { p. } 100 \mathrm{MG} / \mathrm{MS} \text { totale ingérée } \ldots \ldots \ldots \ldots \ldots \ldots \ldots \\
\text { FC total } D M \text { intake }\end{array}$ & $(-0,03 \mathrm{~B})$ & $(-0,04 \mathrm{~B})$ & $\begin{array}{c}1,39 \\
(0,07 \mathrm{~A})\end{array}$ \\
\hline
\end{tabular}

Ce tableau présente les moyennes arithmétiques, et l'estiméc de l'effet des traitements exprimés en déviation par rapport à la moyenne. Les valeurs qui ne portent pas la même lettre minuscule sont différentes au niveau de 5 p. 100 . Celles qui ne portent pas la même lettre majuscule sont différentes au niveau 1 p. 100 . The table shows the arithmetical means, and estimated values of the effect of these treatments, expressed as a deviation relative to the mean. Values which are not followed by the same small letter are significantly different $(P<0.05) ; v a l u e s$ which are not followed by the same capital letter are significantly different $(P<0.01)$. 


\section{TABLEAU 4}

Quantités d'aliments ingérées, production laitière et bilans nutritifs au cours de l'essai 2. Food intake, milk yield and nutritive balance during trial 2.

\begin{tabular}{|c|c|c|c|c|c|c|}
\hline \multirow[b]{2}{*}{$\begin{array}{l}\text { Apport alimentaire de méthionine protégée } \\
\text { Supply of protected methionine }\end{array}$} & \multicolumn{2}{|c|}{$\begin{array}{l}\text { Bas } \\
\text { Low }\end{array}$} & \multicolumn{2}{|c|}{$\begin{array}{l}\text { Haut } \\
\text { High }\end{array}$} & \multicolumn{2}{|c|}{$\begin{array}{l}\text { Analyse } \\
\text { statistique } \\
\text { Statistical } \\
\text { analysis }^{(*)}\end{array}$} \\
\hline & - & + & - & + & $\begin{array}{c}\text { Effet } \\
(\mathrm{H}-\mathrm{B}) \\
\text { Effect } \\
(H-L)\end{array}$ & $\begin{array}{l}\text { Signif. } \\
\text { Signif. }\end{array}$ \\
\hline \multicolumn{7}{|l|}{$\begin{array}{l}\text { Alimentation (kg MS/j) : } \\
\text { Feeding }(\mathrm{kg} \mathrm{DM/d)}:\end{array}$} \\
\hline $\begin{array}{l}\text { Ensilage d'herbe } \ldots \ldots \ldots \ldots \ldots \ldots \ldots \ldots \ldots \ldots \ldots \ldots \\
\text { Maize silage }\end{array}$ & 10,54 & 11,49 & 11,90 & 11,49 & 1,26 & $1 \%$ \\
\hline $\begin{array}{l}\text { Concentré riche en énergie } \ldots \ldots \ldots \ldots \ldots \ldots \ldots \ldots \ldots \\
\text { Energy-rich concentrate }\end{array}$ & 5,23 & 4,04 & 3,83 & 3,84 & & \\
\hline $\begin{array}{l}\text { Concentré riche en azotc } \ldots \ldots \ldots \ldots \ldots \ldots \ldots \ldots \\
\text { Soyabean meal }\end{array}$ & 0,11 & 0,12 & 1,32 & 1,34 & & \\
\hline $\begin{array}{l}\text { Concentré expérimental } \ldots \ldots \ldots \ldots \ldots \ldots \ldots \ldots \ldots \\
\text { Experimental concentrate }\end{array}$ & 0,41 & 0,42 & 0,41 & 0,42 & & \\
\hline $\begin{array}{l}\text { Concentré riche en minéraux } \ldots \ldots \ldots \ldots \ldots \ldots \ldots \ldots \\
\text { Mineral-rich concentrate }\end{array}$ & 0,23 & 0,23 & 0,23 & 0,23 & & \\
\hline $\begin{array}{l}\text { Matière sèche totale ingérée } \ldots \ldots \ldots \ldots \ldots \ldots \ldots \ldots \ldots \ldots \ldots \ldots \ldots \ldots \ldots \ldots \ldots \\
\text { Total dry matter intake } \\
(\mathrm{N} \times 6,25) \text { p. } 100 \mathrm{MS} \ldots \ldots \ldots \ldots \ldots \ldots \\
\text { Crude protein p. } 100 \mathrm{DM}\end{array}$ & 16,52 & 16,28 & 17,70 & 17,31 & 1,54 & $1 \%$ \\
\hline $\begin{array}{l}\text { Production laitière et variation de poids vif : } \\
\text { Milk yield and liveweight variation : }\end{array}$ & & & & & & \\
\hline 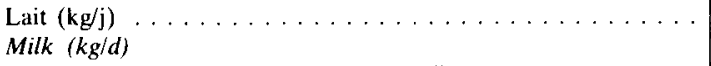 & 22,3 & 20,8 & 24,5 & 24,2 & 2.4 & $1 \%$ \\
\hline $\begin{array}{l}\text { Lait à } 4 \text { p. } 100 \text { de matic̀res grasses }(\mathrm{kg} / \mathrm{j}) \ldots \ldots \ldots \ldots \ldots \\
\text { Fat corrected milk }(F C M-k g / d)\end{array}$ & 22,2 & 20,8 & 24,1 & 23,7 & $? 0$ & $5 \%$ \\
\hline $\begin{array}{l}\text { Taux butyreux }(\mathrm{g} \mathrm{p} .100) \ldots \ldots \ldots \ldots \ldots \ldots \ldots \ldots \ldots \\
\text { Fat content }(g \text { p. } 100)\end{array}$ & 39,3 & 40,1 & 38,4 & 38,6 & -1.2 & $10 \%$ \\
\hline $\begin{array}{l}\text { Taux protéique }(\mathrm{g} \text { p. } 1000) \ldots \ldots \ldots \ldots \ldots \ldots \ldots \ldots \ldots \\
\text { Protein content }(g \text { p. } l 000)\end{array}$ & 28,9 & 29,9 & 29,9 & 30.4 & $1,1)$ & $5 \%$ \\
\hline $\begin{array}{l}\text { Matières grasses }(\mathrm{g} / \mathrm{j}) \ldots \ldots \ldots \ldots \ldots \ldots \ldots \ldots \ldots \ldots \\
\text { Fat }(\mathrm{g} / \mathrm{d})\end{array}$ & 885 & 832 & 954 & 939 & 6 & $5 \%$ \\
\hline $\begin{array}{l}\text { Protéines }(\mathrm{g} / \mathrm{j}) \quad \ldots \ldots \ldots \ldots \ldots \ldots \ldots \ldots \ldots \\
\text { Protein }(\mathrm{g} / \mathrm{d}) \\
\text { Variation du poids vif } \\
\text { (Période expérimentale - Période pré-expérimentale) }(\mathrm{kg}) \\
\text { Liveweight variation } \\
\text { (Experimental - pre-experimental period) }(\mathrm{kg})\end{array}$ & -4 & -1 & 729 & 734 & 94 & $1 \%$ \\
\hline $\begin{array}{l}\text { Apports nutritionnels et efficacité alimentaire : } \\
\text { Nutritive supply and feed efficiency : }\end{array}$ & & & & & & \\
\hline $\begin{array}{l}\text { Apports énergétiques }(\mathrm{UFL} / \mathrm{j}) \ldots \ldots \ldots \ldots \ldots \ldots \ldots \ldots \ldots \\
\text { Energy supply }(F U m / d)\end{array}$ & 14,01 & 13,49 & 15.23 & 14.96 & & \\
\hline $\begin{array}{l}\text { Apports azotés }(\mathrm{g} \text { PDV/j) } \\
\text { Protein supply }(g \text { PDI/d) }\end{array}$ & 1200 & 1165 & 1657 & 1641 & & \\
\hline $\begin{array}{l}\text { Lait } 4 \text { p. } 100 \mathrm{MG} / \mathrm{UFL} \text { disponible pour la production laitière } \\
\text { FCM/FUm for milk yield }\end{array}$ & 2,47 & 2,52 & 2,32 & 2,38 & -0.29 & $5 \%$ \\
\hline $\begin{array}{l}\text { Lait } 4 \text { p. } 100 \mathrm{MG} / \mathrm{MST} \text { ingérée } \ldots \ldots \ldots \ldots \ldots \ldots \ldots \ldots \\
\text { FCM/total } D M \text { intake }\end{array}$ & 1,34 & 1,29 & 1,35 & 1,37 & & N.S. \\
\hline
\end{tabular}

(*) De l'effet du niveau des apports azotes. Of the effect of the level of protein supply.

Le tableau présente les moyennes arithmétiques, et l'estimée de l'effet du niveau des apports azotés. Légendes anglaiscs: voir tablcau 3. The table shows the arithmetical means and the estimated values of the effect of the level of protein supply. English legends : see table 3. 


\section{TABLEAU 5}

Effet du niveau des apports azotés sur la digestibilité de la ration (moyenne et écart-type). Effect of the level of protein supply on the digestibility of the diet (mean and standard deviation).

\begin{tabular}{|c|c|c|}
\hline $\begin{array}{l}\text { Niveau des apports azotés } \\
\text { Level of protein supply }\end{array}$ & $\begin{array}{l}\text { Bas } \\
\text { Low }\end{array}$ & $\begin{array}{l}\text { Haut } \\
\text { High }\end{array}$ \\
\hline \multicolumn{3}{|l|}{ ESSAI $1-T R I A L I$} \\
\hline $\begin{array}{l}\text { Nombre de vaches } \ldots \ldots \ldots \ldots \ldots \ldots \ldots \ldots \ldots \\
\text { Number of cows }\end{array}$ & 5 & 4 \\
\hline $\begin{array}{l}\text { Teneur en MAT }{ }^{(*)} \text { de la ration }(\% \text { MS) } \ldots \ldots \ldots \\
\text { Dietary crude protein content }\end{array}$ & 13,5 & 17,8 \\
\hline $\begin{array}{l}\text { Quantité d’aliments ingérée }(\mathrm{kg} \mathrm{MS}) \\
\text { Total dry matter intake }(\mathrm{kg} / \mathrm{d})\end{array}$ & 13,8 & 13,7 \\
\hline $\begin{array}{l}\text { Digestibilité de la matière organique }(\%)^{(* *)} \\
\text { Organic matter digestibility }(\%)\end{array}$ & $69,4(1,2)$ & $73,5(0,3)$ \\
\hline ESSAI $2-T R I A L 2$ & & \\
\hline $\begin{array}{l}\text { Nombre de vaches } \ldots \ldots \ldots \ldots \ldots \ldots \ldots \ldots \ldots \\
\text { Number of cows }\end{array}$ & 4 & 4 \\
\hline $\begin{array}{l}\text { Teneur en MAT de la ration }(\% \mathrm{MS}) \quad \ldots \ldots \ldots \ldots \\
\text { Dietary crude protein content }\end{array}$ & 13,1 & 17,3 \\
\hline $\begin{array}{l}\text { Quantité d'aliments ingérée }(\mathrm{kg} \mathrm{MS}) \quad \ldots \ldots \ldots \ldots \ldots \\
\text { Total dry matter intake }(\mathrm{kg} / \mathrm{d})\end{array}$ & 16,8 & 16,7 \\
\hline $\begin{array}{l}\text { Digestibilité de la matière organique }(\%) \ldots \ldots \ldots \\
\text { Organic matter digestibility }(\%)\end{array}$ & $70,6(0,5)$ & $71,6(1,0)$ \\
\hline
\end{tabular}

$\left(^{*}\right)$ Matières azotées totales $(\mathrm{N} \times 6,25)$. Crude protein $(N \times 6.25)$.

$\left({ }^{* *}\right)$ Moyennes significativement différentes $(\mathrm{P}<0,05)$. Means significantly different $(P<0.05)$.

Composition des rations (\% MS) / Composition of diets (\% DM) :

Essai 1:

— Ration pauvre en matières azotées: Ensilage de mais : 65,2 ; Concentré énergétique : 28,9 ; Concentré expérimental : 3,1 ; Urée : 1,4 ; Aliment minéral : 1,4 .

- Ration riche en matières azotées : Ensilage de maïs : 66,2; Concentré énergétique : 15,1; Concentré expérimental : 3,2 ; Tourteau de soja : 12,9; Urée : 1,1 ; Aliment minéral : 1,4.

Trial 1:

- Crude protein poor diet : Maize silage : 65.2 ; Energy rich concentrate : 28.9 ; Experimental concentrate: 3.1 ; Urea : $1.4 ;$ Mineral supplement : 1.4 .

- Crude protein rich diet : Maize silage : 66.2 ; Energy rich concentrate : 15.1 ; Experimental concentrate : 3.2 ; Soyabean: 12.9 ; Urea : 1.1 ; Mineral supplement: 1.4 .

Essai 2 :

- Ration pauvre en matières azotées: Ensilage d'herbe : 69,2 ; Concentré énergétique : 26,8 ; Concentré expérimental : 2,6 ; Aliment minéral : 1,4 .

- Ration riche en matières azotées : Ensilage d'herbe : 69,2 ; Concentré énergétique : 19,6 ; Concentré expérimental : 2,6 ; Tourteau de soja - Farine de sang : 7,3; Aliment minéral : 1,4.

Trial 2 :

- Crude protein poor diet : Maize silage : 69.2 ; Energy rich concentrate : 26.8 ; Experimental concentrate : 2.6 ; Mineral complement : 1.4 .

- Crude protein rich diet : Maize silage : 69.2 ; Energy rich concentrate : 19.6 ; Experimental concentrate : 2.6 ; Soyabean meal-blood meal : 7.3 ; Mineral supplement : 1.4 . 
L'augmentation du niveau des apports azotés a entraîné des accroissements de quantité de lait, de taux protéique et de quantité de protéines sécrétées de $0,50 \mathrm{~kg}$, $0,30 \mathrm{~g}$ p. 1000 et $22 \mathrm{~g}$ par point de MAT (p. $100 \mathrm{MS}$ ) supplémentaire en moyenne pour les 2 essais. Elle s'est aussi accompagnée d'une augmentation de la quantité de matières grasses sécrétées d'une quinzaine de grammes par point de MAT (p. 100 MS) supplémentaire, mais on observe une tendance, dans les 2 essais, à la diminution du taux butyreux du lait.

Dans les 2 essais, le poids vif des vaches bien alimentées en azote a légèrement augmenté entre les périodes préexpérimentale et expérimentale, alors qu'il a diminué chez les vaches sous-alimentées, les différences de variations de poids étant de 0,21 et $0,24 \mathrm{~kg}$ par jour dans les essais 1 et 2 respectivement.

Le rapport : lait à 4 p. 100 de matières grasses/UFL disponible pour la production (le calcul de l'apport d'UFL prend en compte les différences de valeur énergétique entre les rations bien pourvues ou déficitaires en azote) a été plus faible dans les lots bien alimentés en azote que dans les lots sous-alimentés. Cependant, si on prend en compte la différence d'évolution du poids vif, il apparaît que la quantité supplémentaire d'énergie sécrétée dans le lait $(0,43 \mathrm{UFL} / \mathrm{kg})$ et déposée dans les tissus corporels ( $5 \mathrm{UFL} / \mathrm{kg}$ ) par les vaches bien alimentées en azote a été 1,52 fois (moyenne des 2 essais) la quantité d'énergie dont elles disposaient en plus à cause de l'accroissement de digestibilité de la ration et de celui des quantités ingérées (essai 2). Des bilans semblables effectués sur les PDI ("Protéines sécrétées en plus dans le lait + protéines déposées en plus dans les tissus corporels »/PDI supplémentaires disponibles), en supposant que le gain de poids vif est constitué de $20 \mathrm{p} .100$ de protéines, conduit à une valeur de 0,37 en moyenne pour les 2 essais. Le rapport : lait à 4 p. 100 de matières grasses/matière sèche totale ingérée a été plus élevé dans le lot enrichi en azote dans l'essai $1(\mathrm{P}<0,01)$, mais il ne l'a pas été, du moins de façon significative, dans l'essai 2.

\section{Discussion et conclusion}

Les $10 \mathrm{~g}$ de $\mathrm{D}, \mathrm{L}$ méthionine protégée qui ont été distribués ont au moins partiellement été absorbés puisque la méthioninémie, faible dans ces essais, a augmenté, comme l'avaient déjà observé RoGers et al. (1987) à la suite d'addition de méthionine protégée à la ration. D'ailleurs, le degré de protection de la $\mathrm{D}, \mathrm{L}$ méthionine protégée utilisée, évaluée avec des moutons équipés de canules du rumen et du duodénum, était d'environ 50 p. 100 (AEC, communication personnelle). L'absence de modification de la production laitière est en accord avec les résultats de récents essais d'alimentation utilisant de la méthionine protégée (dans lesquels, cependant, la teneur en protéines du lait et leur sécrétion étaient parfois augmentées) (YANG et al., 1986 ; CASPER et al., 1987) ; elle corrobore aussi les résultats d'essais nutritionnels dans lesquels des acides aminés étaient infusés dans le tube digestif, après le rumen (cf. Revue de Rulquin \& Champredon, 1987). Cette absence de réponse à la méthionine apportée seule peut être due à certaines de nos conditions expérimentales, a priori défavorables à la manifestation de l'effet de la méthionine : sous-alimentation azotée importante des lots sousalimentés, dans les 2 essais, entraînant probablement une carence multiple en acides aminés indispensables ; utilisation de l'ensilage de maïs dans l'essai 1, dont les protéines 
sont davantage carencées en lysine qu'en méthionine. Cependant, dans le $2^{\mathrm{e}}$ essai réalisé avec des aliments relativement pauvres en méthionine (ensilage d'herbe, farine de sang, tourteau de soja, céréales autres que le maïs) l'apport de méthionine protégée n'a pas non plus modifié la production laitière des animaux. Nos résultats confirment donc ceux à caractère analytique de Rulquin (1987) qui avait observé que l'infusion duodénale de $D, L$ méthionine seule n'avait pas d'effet sur la sécrétion du lait et de ses constituants. Ils n'apportent toutefois aucune explication de l'effet favorable sur la production laitière parfois observé consécutivement à l'apport de méthionine complémentaire dans la ration des ruminants laitiers.

L'accroissement du niveau des apports azotés a entraîné des augmentations de la production (lait, poids vif) des animaux comme l'avaient déjà remarqué de nombreux auteurs (cf. revue de JouRnet et al., 1983a). Elles sont probablement principalement dues aux accroissements concomitants des quantités d'aliments ingérées et de la digestibilité de la ration qui, dans nos 2 essais (en ce qui concerne la digestibilité) comme dans la bibliographie (cf. revue de REMOND, 1985) sont particulièrement fortes avec les régimes à base d'ensilage de maïs. Nos résultats suggèrent également que l'amélioration de l'alimentation azotée a permis une meilleure efficacité de l'énergie dont les animaux disposaient puisque, avec les hypothèses que nous avons prises, le rendement marginal de l'énergie de vaches bien alimentées en azote est supérieur à 1. Une semblable amélioration due à la nutrition azotée n'a toutefois pas été enregistrée par VERMOREL et al. (1982) dans des bilans effectués en chambre respiratoire. Ces résultats confirment cependant l'intérêt d'une bonne alimentation azotée des animaux, qui est particulièrement importante avec les régimes à base d'ensilage de maïs comme l'avaient souligné JOURNET et al. (1983b).

Reçu en août 1988.

Accepté en décembre 1988.

\section{Remerciements}

Nous remercions la Société Alimentation Equilibrée de Commentry (Groupe Rhône Poulenc), 03600 Commentry, qui nous a aidé financièrement dans la réalisation de ces essais et nous a fourni la D,L Méthionine protégée.

\section{Summary \\ Effect of protected methionine supplementation of dairy cow diets : influence of the dietary protein level}

Two trials were carried out during the declining phase of lactation to study the effect of supplementing the diet of dairy cows with $10 \mathrm{~g}$ of $\mathrm{D}, \mathrm{L}$ protected methionine according to the level of protein feeding. In trial 1 ( 9 weeks, of which 3 weeks were preexperimental) the diet based on maize silage, was offered in limited amounts. The 25 primiparous cows used were divided into 3 groups: one group fed a sufficient level of protein and 2 groups fed an insufficient level (17.7 p. 100 and 13.6 p. 100 of crude protein, respectively in the dry matter), one of which received methionine. In trial 2 (13 weeks of which 4 weeks were preexperimental) forage (grass 
silage) was offered ad libitum. The 35 cows were divided into 4 groups according to a $2 \times 2$ factorial design : sufficient or insufficient levels of protein in the diet $(17.5$ p. 100 and 13.6 p. 100 of crude protein respectively in the dry matter) $\times$ supplementation with methionine or not.

Supplementation with protected D,L methionine increased the blood level of this aminoacid but modified neither animal performance nor feed intake (trial 2). Increasing the dietary protein supply, from insufficient levels, led to a higher food intake (trial 2), diet digestibility, milk production and liveweight gain $(0.39 \mathrm{~kg}$ dry matter, 0.60 point of digestibility, $0.50 \mathrm{~kg}$ and $0.05 \mathrm{~kg}$, respectively per point p. 100 of supplementary crude protein (p. 100 DM) in the diet).

Key words : dairy cow, methionine, level of protein feeding, milk, digestibility.

\section{Références bibliographiques}

Casper D.P., Schingoethe D.J., Yang C.M.J., Mueller C.R., 1987. Protected methionine supplementation with extruded blend of soybeans and soybean meal for dairy cows. J. Dairy Sci., 70, 321-330.

Chalupa W., 1975. Rumen bypass and protection of proteins and amino acids. J. Dairy Sci., 58, 1198-1218.

Chandler P.T., Brown C.A., Johnston R.P., Mac Leod G.K., Mc Carthy R.D., Moss B.R., Rakes A.H., SATTER L.D., 1976. Protein and methionine hydroxy analog for lactating cows. J. Dairy Sci., 59, 1897-1909.

Clay A.B., SatTer L.D., 1979. Milk production response to dietary protein and methionine hydroxy analog. J. Dairy Sci., 62 (Supp. 1), 75-76 (Abstr.).

INRA, 1978. Alimentation des ruminants. Ed. INRA Publications (Route de Saint-Cyr), 78000 Versailles.

Jenny B.F., Van Duk H.J., Grimes L.W., O'Dell G.D., 1980. Effect of methionine hydroxy analog at two levels of protein in complete feeds fed to cows in early lactation. J. Dairy Sci., 63 (Supp. 1), 182 (Abstr.).

Journet M., Champredon C., Pion R., Verite R., 1983a. Physiological basis of the protein nutrition of high producing cows. Critical analysis of the allowances. In : IV th Int. Symp. Protein metabolism and nutrition, Ed. INRA Publ., Clermont-Ferrand (France).

Journet M., Faverdin P., Remond B., Verite R., 1983b. Niveau et qualité des apports azotés en début de lactation. Bull. Tech. CRZV Theix, INRA, 51, 7-17.

LundQuist R.G., LinN J.G., OTterby D.E., 1983. Influence of dietary energy and protein on yield and composition of milk from cows fed methionine hydroxy analog. J. Dairy Sci., 66, 475-491.

Mc Carthy R.D., Porter G.A., Griel L.C., 1968. Bovine ketosis and depressed fat test in milk : a problem in methionine metabolism and serum lipoprotein aberration. J. Dairy Sci., 51, 459462.

Norman A.W., Chandler P.T., Park C.S., 1982. The effect of ration protein on milk production response at different stages of lactation. J. Dairy Sci., 65 (Supp. 1), 130 (Abstr.).

Remond B., Champredon C., Decaen C., Pion R., Journet M., 1971. Influence d'un apport de DL-méthionine à des vaches laitières au début de la lactation sur la production laitière et la composition du sang. Ann. Biol. anim. Biochim. Biophys., 11, 455-469.

Remond B., 1985. Influence de l'alimentation sur la composition du lait de vache. 2. Taux protéique : facteurs généraux. Bull. Tech. CRZV Theix, INRA, 62, 53-67.

Rogers J.A., Krishnamoorthy U., Sniffen C.J., 1987. Plasma amino acids and milk protein production by cows fed rumen - protected methionine and lysine. J. Dairy Sci., 70, 789-798.

Rulquin H., 1987. Détermination de certains acides aminés limitants chez la vache laitière par la méthode des administrations post-ruminales. Reprod. Nutr. Dévelop., 27, 299-300.

Rulquin H., Champredon C., 1987. Les acides aminés dans l'alimentation des ruminarts. Bull. Tech. CRZV Theix, INRA, 70, 99-104. 
SEebECK R.M., 1973. The effect of body weight loss on the composition of Brahman Cross and Africander Cross steers. 1. Empty body weight, dressed carcass weight and offal components. J. Agric. Sci. Camb., 80, 201-210.

Stokes M.R., Clark J.H., Steinmetz L.M., 1981. Performance of lactating dairy cows fed methionine or methionine hydroxy analog at two concentrations of dietary crude protein. $J$. Dairy Sci., 64, 1686-1694.

Vermorel M., Remond B., Vernet J., Liamadis D., 1982. Utilization of body reserves by high producing cows in early lactation ; effects of crude protein and amino-acid supply. In : Energy metabolism of Farm Animals. Comptes rendus du $5^{\mathrm{e}}$ Symposium tenu à Lillehamner, Norvège. Publication EAAP $\mathbf{n}^{\circ} 29$. A. Ekern et $\mathbf{F}$. Sundstol Editeurs.

Yang C.M.J., Schingoethe D.J., CASPer D.P., 1986. Protected methionine and heat-treated soybean meal for high producing dairy cows. $J$. Dairy Sci., 69, 2348-2357. 